Mots. Les langages du politique

Les collectivités territoriales en quête d'identité

\title{
Localiser, identifier, valoriser
}

Henri Boyer et Hélène Cardy

\section{OpenEdition}

Journals

Édition électronique

URL : https://journals.openedition.org/mots/20464

DOI : $10.4000 /$ mots. 20464

ISBN : 15/11/2013

ISSN : 1960-6001

Éditeur

ENS Éditions

Édition imprimée

Date de publication : 15 novembre 2011

Pagination : 5-13

ISBN : 978-2-84788-326-8

ISSN : 0243-6450

\section{Référence électronique}

Henri Boyer et Hélène Cardy, «Localiser, identifier, valoriser », Mots. Les langages du politique [En ligne] 97 | 2011, mis en ligne le 15 novembre 2013, consulté le 22 avril 2022. URL : http:// journals.openedition.org/mots/20464 ; DOI : https://doi.org/10.4000/mots.20464 


\section{Localiser, identifier, valoriser}

Pour le spécialiste des questions de langue et de langage, le nom propre qui sert à désigner un lieu, un territoire (le toponyme), relève prioritairement de l'onomastique, discipline qui met en perspective diachronique les noms propres. II reste cependant un fonctionnement du toponyme plus synchronique qui, lui, relève pleinement d'un éclairage sociolinguistique et sociopragmatique ${ }^{1}$. C'est à cet éclairage que l'on voudrait soumettre non seulement la redénomination toponymique à laquelle aspirent certaines collectivités territoriales françaises, mais plus largement leur mise en mots et en scène identitaires, auxquelles elles s'adonnent toutes, singulièrement à l'ère de la décentralisation ${ }^{2} . .$. et de la communication-marketing.

Une précaution tout d'abord. Qu'il s'agisse du nom d'une ville, de celui d'un département ou de celui d'une région, les fonctionnements sociolinguistiquessociopragmatiques ne sont pas identiques et la dimension diachronique, qui n'est jamais absente s'agissant d'un toponyme, pèse d'un poids inégal sur les diverses dénominations concernées. La latitude quant à une éventuelle redénomination n'est pas la même non plus. Ainsi, d'une manière générale, le toponyme

1. Voir le dossier du no 86 (2008) de la revue Mots. Les langages du politique: Toponymes. Instruments et enjeux, coordonné par Henri Boyer et Marie-Anne Paveau.

2. Fondamentalement depuis la première loi de décentralisation (1982). En effet, c'est à cette époque que la communication territoriale, avec ses techniques - balbutiantes - et ses hommes - des «amateurs» -, commence à s'imposer, et c'est autour de la question identitaire que se cristallisent les discours de ceux qui deviendront progressivement des professionnels de la communication. Ces derniers, dès la fin des années soixante-dix, s'emparent des territoires afin de faire évoluer leurs représentations auprès des citoyens, des usagers, des clients et des partenaires économiques par l'intermédiaire d'actions, de supports et de discours adaptés. Ils accompagnent ainsi les mutations de l'espace public, passant, en une trentaine d'années, de discours identitaires à des discours concernant les politiques de «marques » : les territoires sont certes en concurrence en termes d'image (identité), mais aussi en termes de notoriété (économie) et de réputation (innovation, adaptabilité, prise en compte des questions sociétales), et fonctionnent aujourd'hui sur une logique de l'offre et de la demande. L'attractivité territoriale, nouveau credo des professionnels de la communication (mais aussi des élus, des responsables associatifs), ne se présente pas simplement comme un nouveau «discours » sur la façon d'appréhender les territoires, mais comme une nouvelle manière de gouverner et de considérer le citoyen.

Université Montpellier 3, DIPRALANG

henri.boyer@univ-montp3.fr

Université Paris 8, CEMTI

helene.cardy@univ-paris8.fr 
urbain est tellement installé dans le paysage géographique national que son fonctionnement se confond avec l'histoire même du pays et de ses fondations ethnoculturelles3. Ce qui n'est pas le cas avec les départements, dont les dénominations datent pour l'essentiel de la Révolution française et dont le découpage fut arrêté de telle façon qu'il devait être possible d'atteindre le chef-lieu en moins d'une journée de cheval. Une décision finalement plus rationnelle et administrative qu'issue d'une réflexion sur l'homogénéité historique ou la cohérence géographique des territoires en question4. Pour ce qui relève de la toponymie départementale, on sait que ce sont les fleuves (Ariège, Hérault, Rhône...) et les reliefs (Jura, Lozère, Pyrénées) qui en constituent l'ossature. Quant aux régions, leurs toponymes ont été fixés pour la plupart dans les années cinquantesoixante et la référence géographique l'emporte nettement (Auvergne, Centre, Pays de la Loire, Nord - Pas-de-Calais...), même si certains étaient des noms de provinces de l'Ancien Régime (Bretagne, Languedoc, Provence...)5.

Alors qu'en matière d'organisation du territoire national, on prône la centralité, l'unité, l'homogénéité et que l'on fait peu de cas de la différence, de l'hétérogénéité, de la périphérie, sinon pour la gérer en fonction de la perception et des préoccupations du Centre et ainsi l' "aménager », les dénominations toponymiques ne sont fondamentalement que des instruments de localisation. La nomination de l'espace est du reste la fonction cardinale de l'acte de dénomination toponymique, un «acte d'autorité» (Bourdieu, 1982, p.137) qui vise à «faire du territoire» (Akin, 1999, p. 9), même si du «connotatif» est associé au «dénotatif» (Guerrin, 1999, p. 225) pour faire du toponyme plus qu'un toponyme (Boyer, 2008a). On peut aussi bien formuler ce fonctionnement sémiocognitif bivalent parfois ostensible des toponymes en faisant référence aux hypothèses d'Austin, en considérant que, dans ce cas de dénomination topo-

3. Ce qui n'est évidemment pas le cas avec les communautés de communes (CC) et les communautés d'agglomération (CA), de création récente (dernière décennie du $20^{\mathrm{e}}$ siècle), dont les dénominations intègrent certes souvent un toponyme urbain mais en lui associant un autre / d'autres éléments (toponymiques ou autres) qui vise(nt) à singulariser la communauté nouvellement créée. On peut citer pour les CA : Pau-Pyrénées ou Boulogne Côte d'Opale, mais aussi Cœur de Seine (Saint-Cloud, Garches) qui n'utilisent aucun des noms des villes concernées, et à l'inverse, la communauté d'agglomération de La Rochelle, qui ne reprend que le nom de la commune la plus importante. Pour les CC, il était en général difficile de citer les noms des communes concernées, d'où des dénominations toponymiques plus ouvertes: CC du Bocage Champenois, CC des Trois Rivières, CC de la Montagne Bourbonnaise...

4. L'arbitraire relatif de ce découpage administratif (touchant d'ailleurs aussi bien les départements que les régions) est d'ailleurs le fonds de commerce des discours tenus par une très large majorité des directeurs de communication des collectivités territoriales, depuis une trentaine d'années : leur collectivité étant issue d'un découpage administratif hasardeux, ils auraient de ce fait un travail colossal à réaliser pour redonner du sens à ce territoire, pour faire partager un sentiment d'appartenance, créer une cohésion, une identité à cet espace qui en est dépourvu. Les discours sur cette question ont d'ailleurs très peu évolué à l'heure actuelle, ce qui laisse songeur sur l'efficacité des politiques de communication déployées.

5. On sait du reste que les territoires régionaux font depuis les origines l'objet de contestations, de nature différente. 
nymique, à une orientation illocutoire - pour paraphraser Austin : le toponyme localise EN nommant - s'adjoint une orientation perlocutoire - le toponyme marque une identité PAR le fait de nommer (Austin, 1970).

Ce supplément «connotatif», qui exploite la dimension perlocutoire, est de nature variable. Il peut être d'origine historico-culturelle (Provence, Aquitaine par exemple) et dans ce cas, il est le plus souvent au principe même de l'acte de dénomination toponymique. Mais il peut être dû à l'impact de faits historiques, de rumeurs, de changements plus ou moins importants dans la société. On peut dire que la traduction en est une plus-value identitaire (négative/ positive) et au fil des siècles ou même des décennies, le toponyme peut être soumis à un processus de figement représentationnel du fonctionnement bivalent (localisant-identifiant), de patrimonialisation pourrait-on dire, qui en font une unité compacte de l'imaginaire ethnosocioculturel collectif. Il en va ainsi par exemple pour Marseille, l'Alsace et la Lorraine, la Corse... Mais il est évident que cette dimension connotative à teneur identitaire peut être due à des facteurs beaucoup plus conjoncturels car en rapport avec de nouvelles donnes politico-administratives (comme la régionalisation), touristiques ou liées à l'air du temps. C'est évidemment alors la stratégie socio-pragmatique ostensible qui vient corriger ce qui est vécu comme un déni d'identité ou parfois une dévalorisation identitaire ou plus simplement une absence totale de connotation identitaire. L'impératif communicationnel pousse à travailler le signifiant toponymique conformément au signifié que l'on souhaite promouvoir'. À l'ère des "dircoms», les politiques de communication institutionnelle des collectivités territoriales, par l'intermédiaire de discours visibles sur des supports qui se multiplient (des plus traditionnels aux sites web, plateformes, etc.), ont eu à cœur de créer de l'identité ou de corriger une identité mal vécue7. Il en est ainsi de ce qui est ressenti comme dévalorisé, en particulier ce qui est «bas» : Saint-Geniès-le-Bas, dans l'Hérault, a pu être ainsi renommé Saint-Geniès-deFontedit; de même, les Basses-Alpes sont devenues Alpes-de-Haute-Provence.

Ce sont autant d'interventions qui concourent à produire de la « plus-value toponymique» (Guerrin, 1999, p. 225) de nature identitaire. Dans la présente livraison de Mots. Les langages du politique, plusieurs cas sont étudiés qui montrent le développement croissant de ces stratégies, en particulier des campagnes de promotion des territoires qui tentent de faire flèche de tout bois. On songe à l'exploitation des nouvelles valeurs écologiques, par exemple. On songe également à la mise en évidence de caractéristiques culturelles et/ou patrimoniales de certains territoires, comme la Grotte Chauvet pour le département de l'Ardèche. Mais il existe aussi, on l'a vu, des stratégies institutionnelles

6. On est alors dans l'ordre du performatif, de l'action, qui vise à faire advenir une réalité ou à modifier l'existant (Austin, 1970). Voir également Akin, 1999 (p.59) qui souligne cette « performativité».

7. Illustrant ainsi le principe selon lequel «l'identité n'est pas plus un donné qu'une essence : c'est le résultat d'une production signifiante» (Lafont, 1986, p. 8). Voir également Boyer, 2008b. 
qui cherchent purement et simplement à revoir la dénomination toponymique en fonction de la même visée identitaire. C'est le cas pour les projets (en nombre certes limité) de redénomination, plus ou moins récents, avortés ou aboutis ${ }^{8}$. Il en est ainsi de la redénomination de certains départements, comme les Côtes-du-Nord devenues en 1990 Côtes-d'Armor après une longue procédure. Ce cas est intéressant car la redénomination était parfaitement légitimée sur le plan dénotatif : elle corrigeait avant tout un déficit de localisation, mais elle introduisait en même temps une plus-value identitaire (en marquant l'appartenance à l'aire celtique).

On pense également aux entreprises de redénomination frustrées de la région Provence - Alpes - Côte d’Azur, nommée plus lapidairement PACA, ou encore de celle de Languedoc-Roussillon. Ces deux projets non aboutis méritent une attention particulière, l'interruption n'étant certes pas dans les deux cas de même nature, mais révélant cependant des phénomènes intéressants quant au fonctionnement toponymique bivalent dont il a été question, au pouvoir de renommer... et à ses limites.

Dans le cas du projet de redénomination de la Région PACA, c'est le président du conseil régional lui-même, Michel Vauzelle, qui en est à l'origine. En juin 2009, après avoir considéré publiquement que «PACA, franchement, c'est très, très laid », que le toponyme complet était «trop long et peu pratique » ( $L$ 'Express, 8 octobre 2009) et qu'il était ainsi inévitablement réduit à l'acronyme $P A C A$, il décidait de lancer une consultation auprès des habitants de la région, entre le 11 et le 31 juillet 2009, par Internet et par courrier, sous le titre : «Quel nom pour notre région?» Plus de sept mille personnes (7724) ont participé à la consultation et 10102 réponses ont été récoltées, qui ont permis de dégager 1667 "propositions uniques» (les consultés pouvant formuler jusqu'à trois propositions). Un comité d'experts9 a été chargé, à partir des propositions recueillies, de sélectionner quelques redénominations. Il s'agit du reste des sept noms ayant fait les scores les plus importants: Provence, Pays de Provence, Provence-Méditerranée, Alpes-Méditerranée, AlpesProvence, Provence-Alpes-Méditerranée et Provence - Alpes - Côte d'Azur..., la première et la dernière proposition (qui choisit le maintien de la dénomination en vigueur) obtenant les deux scores les plus élevés (2424 pour la première et 1283 pour la dernière). Il est clair que les choix majoritaires se sont portés sur de nouveaux toponymes à forte pertinence localisante (difficile cependant à

8. C'est le cas aussi pour les stratégies de communication qui visent, avec plus ou moins de succès, à imposer une image valorisante de certaines villes par l'usage d'une qualification après un toponyme non marqué en la matière. Georges Frêche, alors maire de Montpellier, avait promu «Montpellier la surdouée» (1984), slogan qui n’a pas trop mal réussi, si l'on considère les succès enregistrés dans la perception nationale, voire internationale, de la ville au cours des deux dernières décennies du $20^{\mathrm{e}}$ siècle.

9. Composé de personnalités universitaires, de conseillers régionaux, de membres du CESR et de conseillers régionaux jeunes. 
préserver face à Provence - Alpes - Côte d'Azur...) même si Provence (ou Pays de Provence), bien qu'avec une pertinence localisante inférieure, est incontestablement investi d'une teneur identitaire forte.

Une consultation populaire, dernière phase de l'opération de redénomination, était programmée après les élections régionales de mars 2010. Malgré, semble-t-il, l'implication sincère du président Vauzelle, la crise économique a eu (officiellement) raison de la finalisation du projet ${ }^{10}$ : il eût été inconvenant, auxyeux de l'opinion publique régionale, de réaliser la dernière phase de l'opération, dont le coût menaçait d'être très élevé. La consultation populaire est donc remise à plus tard, si le contexte redevient favorable ${ }^{11}$.

Un deuxième échec de redénomination, dont la raison est bien différente, concerne la région Languedoc-Roussillon et la volonté frustrée de Georges Frêche, alors président du conseil régional, de donner à sa région le nouveau nom de Septimanie (Boyer, 2008a, p.12-17).

En 2004, une majorité d'élus de gauche porte à la tête du nouveau conseil régional l'ex-maire de Montpellier, Georges Frêche. Celui-ci affiche, avec l'énergie qui lui a si bien réussi pour changer la ville et son image, sa volonté de rompre avec l'ère précédente, qui avait vu la droite aidée de l'extrême-droite présider aux destinées de la région. Les initiatives susceptibles d'incarner la rupture en question n'ont pas manqué, mais c'est la volonté du nouveau président de changer la dénomination de la région Languedoc-Roussillon au profit d'un toponyme inédit qui a surpris. La dénomination en vigueur avait été le fruit, en 1960, d'un compromis obtenu par la reconnaissance de la nature biculturelle de la région ${ }^{12}$ : quatre départements sur cinq appartenant à l'aire languedocienne, un cinquième (les Pyrénées-Orientales) appartenant à l'aire catalane. Le composé Languedoc-Roussillon prenait acte de cette double identité et permettait à Perpignan d'accepter la tutelle de Montpellier (au lieu de celle de Toulouse). C'est dire si le projet frêchien était périlleux, beaucoup plus, semblet-il, que ne l'avait pressenti le nouveau président. Néanmoins Georges Frêche, comme à l'accoutumée, battit la campagne avec une stratégie de communication dispendieuse en faveur de la labellisation de produits agroalimentaires régionaux visant à imposer, en relation avec un argumentaire à caractère historique bien senti (mais somme toute peu convaincant) le nouveau toponyme

10. Cependant les résultats de la première phase ont été diffusés en 2009 par le conseil régional. À noter que la région Centre avait entamé une procédure similaire, avec un mode de consultation semblable, au milieu des années quatre-vingt-dix. Un nouveau nom pour la région avait finalement été proposé (Centre Val de Loire). Le changement de nom d'une région devant faire l'objet d'un projet de loi, il semble qu'au bout du processus, le dossier ait été enterré.

11. Nous remercions M. Pierrel, chargé de mission auprès du président de la région PACA, pour l'entretien qu'il a accordé à Henri Boyer en janvier 2011, au cours duquel il a livré une information de première main concernant l'entreprise de redénomination décidée par M. Vauzelle, ainsi que pour les documents qu'il a communiqués, dont le Rapport du comité d'experts.

12. Bernard Rieu, L'Indépendant, 27 juillet 2005. 
Septimanie. C'était sans compter sur une réaction de ses opposants politiques, certes, mais aussi et surtout, semble-t-il, sur l'exaspération et la mobilisation des citoyens, surtout ceux du Roussillon, aidés de manière efficace par les quotidiens Midi Libre et L'Indépendant qui ont ouvert leurs colonnes au refus collectif de la redénomination programmée. Georges Frêche, accusé de perpétrer un coup de force, eut la sagesse politique de faire marche arrière et d'enterrer son projet de redénomination, mettant ainsi un terme à une polémique qui menaçait ses bases politiques. Il est évident que cette péripétie de la vie politique régionale révèle une donnée de base qui intéresse notre propos ici : la dénomination d'une région (ou d'un département), dans l'hypothèse où a pesé sur sa genèse une préoccupation identitaire, subit immanquablement un processus de patrimonialisation qui la rend sinon intouchable, du moins résistante à toute velléité de redénomination.

Si la question de l'identité est structurante depuis une trentaine d'années dans le travail des chargés de communication, on constate néanmoins une évolution dans les objectifs affichés (d'une communication «descendante» à visée identitaire à l'émergence d'un «marketing territorial », en passant par une communication de «proximité»), les supports utilisés (les médias les plus traditionnels - journal interne, plaquettes, affiches... - trouvant un relais efficace dans les NTIC et leur développement - sites web, forums, plateformes collaboratives) et les pratiques qui encouragent la participation (développement de l'e-administration, conseils de quartier...). Depuis une dizaine d'années en particulier, sont adaptées aux territoires des stratégies dont les termes sont pour partie empruntés au monde de l'entreprise ${ }^{13}$. La question de l'image de marque des collectivités est devenue centrale, accompagnée d'une nébuleuse de termes qui renvoient eux-mêmes à des logiques «marchandes»: marque, attractivité, compétitivité, innovation, créativité... Les rapports entre les élus et les citoyens se sont inversés, passant d'une logique de l'offre à une logique de la demande : à l'ère du marketing territorial (les territoires cherchent à se valoriser pour acquérir puis conserver leurs cibles, aussi diverses soient-elles, par des offres suffisamment attractives face à la concurrence), le citoyen est devenu un client, voire un partenaire, dont il faut tenir compte.

Comment se démarquer? Comment devenir et rester un territoire attractif? Comment faire adhérer la population à un discours censé produire des signes distinctifs, qui mettent en valeur un territoire? Si l'enjeu économique est évident (il faut attirer à la fois des investisseurs et des touristes et maintenir une population sur son territoire), il existe bien d'autres enjeux, symboliques et politiques.

13. Voir le $17^{\mathrm{e}}$ colloque du CECCOPOP (Centre d'études comparées en communication politique et publique) : «Marketing territorial et marketing des entreprises, qui inspire l'autre? Réseaux sociaux, politiques de marques, techniques d'influence », 24 juin 2011, Paris. 
Les contributions à ce dossier tentent de répondre à ces questions sous plusieurs angles, en faisant éventuellement apparaître les tensions qui existent lorsque l'on doit mettre en mots (et en scène) un territoire.

Celle de Marie-Ange Grégory s'intéresse aux redénominations des départements (la Charente-Maritime, les Alpes-de-Haute-Provence, les Côtes-d'Armor) qui, à la différence de l'immense majorité de ces collectivités territoriales consacrées par la Révolution, ont changé de toponyme au cours du $20^{e}$ siècle. L'auteure se propose d'observer les discours tenus lors de ces processus de redénomination pour en analyser les logiques argumentatives ainsi que les ressorts identitaires et économiques. Elle montre que «l'inexactitude géographique et la valeur péjorative» sont bien les arguments les plus invoqués. Mais les projets de changement de toponyme doivent être acceptés localement et nationalement et neutraliser obstacles et objections pour parvenir à s'imposer. L'article montre que la détermination inlassable des acteurs concernés est évidemment décisive.

La redénomination est également au cœur de l'article de Christian Le Bart et Thomas Procureur qui focalisent leur analyse sur l'un des cas évoqués dans la contribution précédente : le département des Côtes-du-Nord qui, en 1990, est devenu le département des Côtes-d'Armor. Selon les auteurs, la décentralisation a représenté pour les collectivités territoriales une nouvelle donne qui a instauré une «logique de marketing» dans laquelle le toponyme « [est devenu] un élément clé de l'attractivité [du territoire] » : le processus de redénomination abouti des Côtes-du-Nord en Côtes-d'Armor en témoigne.

Parmi les traits identitaires mis en discours par les régions, la/les langue(s) présente(s) sur le territoire (avec plus ou moins de vitalité) a/ont bien évidemment une place de choix. Le cas de la région Rhône-Alpes, où cohabitent le franco-provençal et l'occitan, est traité dans ce dossier pas Michel Bert et James Costa. Ces auteurs observent l' «entrée sur la scène politique régionale» des deux langues de la région, au travers de la construction du discours institutionnel produit par le conseil régional. Des enquêtes commandées par l'institution, et visant à apprécier la pratique des langues au sein du territoire régional, ont servi de levier à une politique visant la «valorisation d'un patrimoine linguistique » mais aussi la promotion des usages de l'occitan et du franco-provençal, en situation de faiblesse comme les autres langues régionales de France.

S'intéressant aux «petites villes» à travers l'étude des images et des discours utilisés sur leurs sites Internet, Hélène Mainet montre leur difficulté de positionnement et le délicat travail de mise en place des actions de marketing. Bien que celles-ci développent, surtout les plus importantes d'entre elles en termes de population, de véritables stratégies de communication pourvaloriser leurs atouts, l'auteur montre toute la difficulté qu'elles éprouvent à se trouver un discours à la fois propre et tout à fait adapté, qui soit en mesure de rendre compte de la réalité de leur situation. La communication de ces petites villes 
s’organise immanquablement autour de références aussi bien à leur urbanité qu'à leur ruralité, et ce quelle que soit leur taille : elles se comparent systématiquement aux autres niveaux territoriaux, soit pour s'en rapprocher, soit pour s'en démarquer.

Maïa Drouard, quant à elle, s’intéresse au processus de patrimonialisation initié par les collectivités territoriales à la suite de la découverte de la Grotte Chauvet. Elle met en évidence la façon dont les discours se sont organisés autour du projet de la création de l'Espace de restitution de la Grotte Chauvet (ERGC), conçu comme un objet culturel. Par l'intermédiaire d'un processus pédagogique, les élus y voient une opportunité d'élaborer une toute nouvelle image pour le département de l'Ardèche, à la fois dans l'optique d'une appropriation de ce patrimoine à des fins de restructuration du tourisme, mais aussi par la population (création d'une identité commune et renforcement de l'identité départementale). En effet, une grande partie de l'acceptation du dossier demandé pour le classement de la grotte au patrimoine mondial de l'Unesco repose sur la façon dont la population s'approprie ce patrimoine. Et la labellisation est essentielle pour asseoir la légitimité du projet.

Il est également question de labellisation dans le travail proposé par Hélène Cardy, qui questionne la forme du palmarès comme nouvel outil au service de stratégies de communication et de valorisation du territoire. Elle souhaite montrer en quoi la dimension médiatique de ces évaluations contribue au renforcement ou parfois à la (re)construction des images liées à un territoire. Mais d'autres éléments entrent en jeu. Ainsi on s'interroge sur la façon dont les discours de politiques publiques (ceux liés aux nouvelles technologies et à l'environnement en particulier) pourraient imprégner l'élaboration de ces prix destinés aux collectivités territoriales, contraignant ainsi les porteurs de projet (chargés de communication, élus) à modifier leurs pratiques pour être «médaillables». On montre également comment ces palmarès peuvent se présenter comme des outils de légitimation du travail des instances en charge de la communication mais aussi quels peuvent être les enjeux de la labellisation par rapport à la mise en compétition accrue des territoires, et les effets possibles de la médiatisation de ces palmarès.

Au-delà de la question de la redénomination de certains territoires, que l'air du temps semble priser et qui montre la sensibilité particulière du politique en la matière, ce dossier souhaite mettre en évidence la dimension sociopragmatique des discours officiels tenus et les stratégies de mises en scène identitaires programmées depuis les débuts de la décentralisation par les instances de communication institutionnelles des collectivités concernées : communes, départements, régions. 


\section{Références}

AkIN Salih, 1999, "Pour une typologie des processus redénominatifs», Noms et renoms. La dénomination des personnes, des populations, des langues et des territoires, S. Akin éd., Rouen, Publications de l'université de Rouen.

Austin John L., 1970, Quand dire, c'est faire, Paris, Seuil.

Bourdieu Pierre, 1982, Ce que parlerveut dire, Paris, Fayard.

BOYER Henri, 2008a, «Fonctionnements sociolinguistiques de la dénomination toponymique », Mots. Les langages du politique, n 86, mars, p 9-21.

- 2008b, Langue et identité, Limoges, Lambert-Lucas.

GUERRIN Christian, 1999, «Les processus redénominatifs dans les noms de communes françaises depuis 1943. Étude socio-toponymique de la variation dans la nomenclature administrative», Noms et re-noms. La dénomination des personnes, des populations, des langues et des territoires, S. Akin éd., Rouen, Publications de l'université de Rouen.

LAFOnT Robert, 1986, «Contrôle d'identités », dans La Production d'identités, Montpellier, Université Paul-Valéry, CNRS. 